\title{
Development of $\mathrm{MoS}_{2}$ Nanosheets Embedded Nickel Composite Coating and its Mechanical Properties
}

Antony Joseph, Mugilan Narayanasamy, Balakrishnan Kirubasankar and Subramania Angaiah"

$\mathrm{MoS}_{2}$ nanosheets were prepared by a facile hydrothermal method and its morphology and structural properties were characterized by FE-SEM and XRD analysis. $\mathrm{MoS}_{2}$ nanosheets reinforced $\mathrm{Ni}$ composite coatings were prepared by a simple electrodeposition method using various concentrations of $\mathrm{MoS}_{2}$ in Watts nickel plating bath. Surface morphology, microstructure and crystal orientation of pure Ni and Ni-MoS composite coatings were characterized by FE-SEM and XRD analysis. Microhardness and wear resistance of pure Ni coating were improved by the addition of $\mathrm{MoS}_{2}$ nanosheets in the Ni matrix to use as solid lubricant.

Keywords: Electrodeposition; Ni-MoS 2 composite Coating; $\mathrm{MoS}_{2}$ Nanosheets; Liquid exfoliation; Microhardness

Received 30 August 2018, Accepted 28 October 2018

DOI: $10.30919 / \mathrm{esmm} 5 \mathrm{f} 152$

\section{Introduction}

Electrodeposition is a simple and efficient method to produce metallic coatings with improved surface properties such as hardness, wear resistance and corrosion resistance. ${ }^{1}$ Nickel is known to be a good electrodeposited metal because of its high mechanical properties and compatibility with most of the substrate materials. Electrodeposited Ni has high density, excellent wear and corrosion resistance and potential substitute for hard chromium coatings. As the grain size of the $\mathrm{Ni}$ reduces, the strength and strain hardening rate also increases. Electrodeposited nickel and nickel composites are emerging as a good replacement for environmentally harmful and carcinogenic hexavalent hard chrome coating. ${ }^{2-6}$ Electrodeposited metal matrix nanocomposite coatings exhibit enhanced material properties such as microhardness, wear and corrosion resistance than the pure metal coatings. These enhanced properties depend mainly on the nature of deposited nanofillers and the distribution of nanofillers in the metallic matrix. However, it is difficult to achieve a stable homogenous dispersion of deposited fillers in the metallic matrix. Several researchers have deposited different nanofillers such as transition metal oxides, rare earth metal oxides, and carbon-based materials embedded nickel composite coatings to enhance the microhardness, wear, and corrosion resistance. ${ }^{7-16}$ Venkatesh et al. studied the mechanical and corrosion properties of Ni-Graphene nanocomposite coating and an achieved improved microhardness and corrosion resistance than pure Ni coating. ${ }^{17}$

After the successful exfoliation of graphene, 2D-materials have been received much attention. Transition metal dichalcogenides (TMDs) such as $\mathrm{MoS}_{2}, \mathrm{MoSe}_{2}, \mathrm{WS}_{2}, \mathrm{Wse}_{2}, \mathrm{WTe}_{2}$ etc are important 2D materials and alternatives for graphene in semiconducting applications due to their large intrinsic bandgap. ${ }^{18}$ Molybdenum disulfide $\left(\mathrm{MoS}_{2}\right)$ has Mo layer sandwiched between two layers of S (S-Mo-S) and stacked into a 3D arrangement by weak Van der Waals forces. Such layered

Electro-Materials Research Laboratory, Centre for Nanoscience and Technology, Pondicherry University, Puducherry - 605014, India

*E-mail: a.subramania@gmail.com arrangements allow the $\mathrm{MoS}_{2}$ layers to easily shear between the basal planes and are responsible for the excellent lubricity of $\mathrm{MoS}_{2}$ and thereby utilized as a solid lubricant. The in-plane stability of graphene is responsible for its extraordinary mechanical properties which are utilized in metal matrix composites. This in-plane stable structure is common for all other 2D-materials. But other 2D-materials, especially $\mathrm{MoS}_{2}$ is underutilized for reinforcement purposes. Previous researches have shown that $2 \mathrm{D}-\mathrm{MoS}_{2}$ possess high mechanical properties than their bulk form. Castellano-Gomez et al. measured the elastic properties of freely suspended few-layer $\mathrm{MoS}_{2}$ nanosheets (5 to 25 layers) in a bending test performed using AFM. The Young's modulus value for $\mathrm{MoS}_{2}$ nanosheets obtained from the experiment is $E=0.33 \pm 0.07 \mathrm{TPa}$, which is very high and only one third lower than exfoliated graphene (E $=0.8-1.0 \mathrm{TPa})$, which is higher than graphene oxide $(0.2 \mathrm{TPa})$ and bulk $\mathrm{MoS}_{2}(0.24 \mathrm{TPa})$. These results showed that few-layers $\mathrm{MoS}_{2}$ can be effectively used as a reinforcement for metal matrix composites and as an alternative for graphene. ${ }^{19}$ Recently, $\mathrm{He}$ et al. prepared $\mathrm{MoS}_{2}$ nanoparticles embedded Ni-P composite coating and studied its mechanical and wear resistance properties. ${ }^{20}$ But, there are no reports on $\mathrm{MoS}_{2}$ nanosheets embedded nickel composite coatings. Hence, in the present investigation, we prepared few layers of $\mathrm{MoS}_{2}$ nanosheets by hydrothermal method and codeposited with $\mathrm{Ni}$ matrix by simple electrodeposition technique. Its microhardness and wear resistance are studied in detail and compared with $\mathrm{MoS}_{2}$ nanoparticles embedded $\mathrm{Ni}$ composite and pure Ni coatings.

\section{Experimental}

Chemicals and reagents - All the chemicals and reagents were extra pure. Ammonium molybdate $\left.\left(\mathrm{NH}_{4}\right)_{6} \mathrm{Mo}_{7} \mathrm{O}_{24}\right)$, thiourea $\left(\mathrm{CH}_{4} \mathrm{~N}_{2} \mathrm{~S}\right)$, boric acid and sodium dodecyl sulphate were obtained from Merck, India. Nickel sulphate and nickel chloride were purchased from Hi-media, India. Deionized water is used throughout the process. All chemical and reagents were of analytical grade

Synthesis of $\mathrm{MoS}_{2}$ nanosheets - $\mathrm{MoS}_{2}$ nanosheets were prepared by a facile hydrothermal method using ammonium molybdate $\left(\mathrm{NH}_{4}\right)_{6}$ $\mathrm{Mo}_{7} \mathrm{O}_{24}$ ) and thiourea as the Mo and S precursors, respectively. $4.96 \mathrm{~g}$ of 
$\left(\mathrm{NH}_{4}\right)_{6} \quad \mathrm{Mo}_{7} \mathrm{O}_{24}$ and $9.12 \mathrm{~g}$ of thiourea were dissolved in $150 \mathrm{ml}$ deionized water and stirred for $30 \mathrm{~min}$. This solution was transferred into a $200 \mathrm{~mL}$ Teflon-lined stainless steel autoclave, sealed and is heated in an oven at $220{ }^{\circ} \mathrm{C}$ for $24 \mathrm{~h}$ and then allowed to cool down to room temperature. The product was then collected, centrifuged, washed with water and ethanol and dried in vacuum oven at $70{ }^{\circ} \mathrm{C}$ for $12 \mathrm{~h}$.

Characterization of $\mathrm{MoS}_{2}$ nanosheets - The surface morphology of prepared $\mathrm{MoS}_{2}$ nanosheets were analyzed by FE-SEM (JSM, JEOL Model: $7600 \mathrm{~F}$ ) analysis. X-ray diffraction analysis was carried out to confirm the formation of few layer $\mathrm{MoS}_{2}$. XRD pattern was recorded using X-ray diffractometer (Rigaku, Ultima IV, Japan) with $\mathrm{Cu}-\mathrm{K} \alpha$ radiation $(\lambda=1.54 \AA)$ and its $2 \theta$ value ranging from $10^{\circ}$ to $80^{\circ}$.

Electrodeposition of $\mathrm{Ni}-\mathrm{MoS}_{2}$ composite coatings - Electrodeposition of pure $\mathrm{Ni}$ and $\mathrm{Ni}-\mathrm{MoS}_{2}$ nanocomposite coatings were carried out by direct current on mild steel substrates $\left(2.5 \times 2.5 \mathrm{~cm}^{2}\right)$ using Watt's nickel bath. The bath composition and electrodeposition parameters are given in Table 1. Nickel metal and mild steel substrate were used as the anode and cathode, respectively. Prior to plating, the mild steel substrates were mechanically polished using different grade emery papers, degreased with acetone, cleaned with soap solution and rinsed with distilled water. They were then dipped in $10 \% \mathrm{H}_{2} \mathrm{SO}_{4}$ solution for $10 \mathrm{sec}$ and immediately transferred to the plating bath. $\mathrm{MoS}_{2}$ nanosheets were dispersed in the plating bath to obtain Ni-MoS 2 composite coating. Deposition was carried out by the addition of different concentrations of $\mathrm{MoS}_{2}$ in the bath and also by varying the stirring rate of the bath. Prior to electrodeposition, the bath solution was stirred using magnetic stirrer at $500 \mathrm{rpm}$ for $24 \mathrm{~h}$ and subsequently ultrasonicated for $15 \mathrm{~min}$ to ensure a uniform dispersion of nanosheets in the plating bath.

Characterization of Ni-MoS 2 composite coatings - The XRD and FE-SEM studies were done on pure $\mathrm{Ni}$ and $\mathrm{Ni}-\mathrm{MoS}_{2}$ composite coating with an optimized concentration of $\mathrm{MoS}_{2}$ and stirring rate of $1 \mathrm{~g} / \mathrm{L}$ and $400 \mathrm{rpm}$, respectively. The surface morphology for pure $\mathrm{Ni}$ and $\mathrm{Ni}$ $\mathrm{MoS}_{2}$ composite coating were analyzed using Field Emission Scanning Electron Microscope (JSM, JEOL Model: 7600F). XRD pattern of the coatings were recorded using X-ray diffractometer (Rigaku, Ultima IV, Japan) with $\lambda_{\mathrm{CuKa}}=1.54 \AA$ and $2 \theta$ ranging from $10^{\circ}$ to $80^{\circ}$ to study the phase purity and crystal structure. The average crystallite size of nickel and nickel composite coatings were calculated using the following Scherrer equation (Eq. 1);

$$
D=\frac{K \lambda}{\beta \cos \theta}
$$

where $\mathrm{D}$ is the average crystallite size, $\mathrm{K}$ is the shape factor (typically $0.89), \lambda$ is the wavelength of incident $\mathrm{X}$-ray radiation, $\beta$ is the full width half maxima (FWHM) or the peak width (radians) at half of the maximum intensity and $\theta$ is the Bragg's angle (degree). The preferred crystal orientation of the coatings were calculated from the diffractogram data using the relative texture coefficient (RTC) relation given in Eq. 2.

$$
R \mathrm{TC}=\frac{I_{h k l} / I_{h k l}^{O}}{\sum\left(I_{h k l} / I_{h k l}^{o}\right)} \times 100
$$

where, $I_{h k l}$ and $I_{h k l}$ are the diffraction intensity of (hkl) planes of the coating and the standard Ni powder sample, respectively.

Williamson-Hall method from the line broadening in XRD pattern was used to confirm the crystallite size of $\mathrm{Ni}$ and to identify the lattice strain during electrodeposition. ${ }^{21}$ Broadening of X-ray diffraction peaks is mainly due to three factors: instrumental broadening, crystallite size, and lattice strain. In this method, it is assumed that the X-ray diffraction peak is a convolution of Lorentzian curve (influence of grain size) and Gaussian curve (broadening due to the strain). ${ }^{22}$ Whereas, instrumental broadening error is not considered. Williamson and Hall suggested a relation for the FWHM of the profile which can be expressed as follows;

$$
\beta \cos \theta=\frac{K \lambda}{D}+4 \varepsilon \sin \theta
$$

where, $D$ and $\varepsilon$ are volume weighted average crystallite size and lattice strain, respectively. Williamson-Hall plot is drawn between $4 \sin \theta$ at the $\mathrm{x}$-axis and $\beta \cos \theta$ at the $\mathrm{y}$-axis. The slope and $\mathrm{y}$-intercept of the plot give the values of lattice strain and crystallite size, respectively.

Microhardness of the coatings were analyzed using Vickers microhardness tester (Wilson Wolpert, Germany) at a load of $0.5 \mathrm{Kg}$ for a dwell time of $20 \mathrm{sec}$. Friction measurements were carried out by employing pin (8 $\mathrm{mm}$ diameter) on disc (high carbon high chromium steel) tribometer (Ducom instruments, India) at $5 \mathrm{~N}$ load with a sliding velocity of $0.5 \mathrm{~m} / \mathrm{s}$.

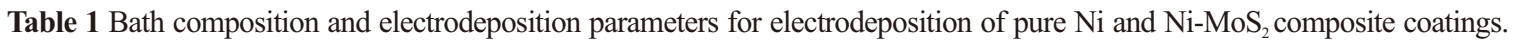

\begin{tabular}{lr}
\hline & Bath composition \\
\hline Nickel sulphate & $240 \mathrm{~g} / \mathrm{L}$ \\
Nickel chloride & $20 \mathrm{~g} / \mathrm{L}$ \\
Boric acid & $40 \mathrm{~g} / \mathrm{L}$ \\
Sodium dodecyl sulphate & $0.1 \mathrm{~g} / \mathrm{L}$ \\
MoS $_{2}$ nanosheets & Electrodeposition parameters \\
\hline Current density & $0.5,1.0,1.5 \mathrm{~g} / \mathrm{L}$ \\
pH & \\
Temperature & $2.5 \mathrm{~A} / \mathrm{dm}^{2}$ \\
Plating time & $\sim 4.5$ \\
Agitation & $\sim 50^{\circ} \mathrm{C}$ \\
\hline
\end{tabular}




\section{Results and discussion}

Characterization of $\mathrm{MoS}_{2}$ nanosheets - The XRD pattern of prepared $\mathrm{MoS}_{2}$ nanosheets is shown in Fig.1a. The four distinct and clear peaks having planes (002), (100), (103) and (110) can be indexed to the standard powder diffraction file of hexagonal phase of bulk $\mathrm{MoS}_{2}$ (JCPDS 37-1492). No other characteristic impurity peaks are found indicating that the prepared $\mathrm{MoS}_{2}$ nanosheets are highly pure. The broad peaks suggest that the crystallite size was at the nanolevel. The most prominent peak having the (002) plane shows a shift towards the lower angle with a $2 \theta$ value of $14.05^{\circ}$ which is attributed to the lattice expansion and introduction of strain. ${ }^{23}$ The interlayer distance has been calculated using the Bragg's equation and is found to be $6.30 \AA$ which is greater than the bulk $\operatorname{MoS}_{2}(6.15 \AA)$ having lattice parameters, $a=$ $3.15 \AA$ and $\mathrm{c}=12.30 \AA .^{24}$ The increase in interlayer spacing confirms the formation of few layers of $\mathrm{MoS}_{2}$ nanosheets. FE-SEM image reveals that $\mathrm{MoS}_{2}$ is exfoliated into few layers of nanosheets [Fig.1b].

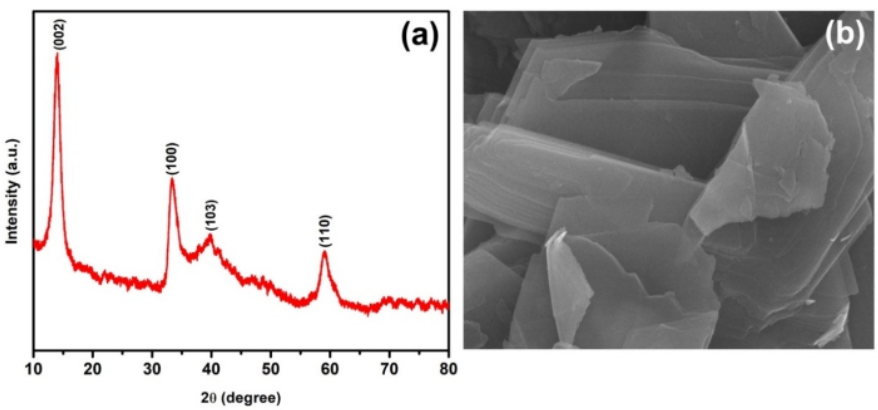

Fig. 1 (a) XRD pattern of $\mathrm{MoS}_{2}$, (b) FE-SEM photograph of few layer of $\mathrm{MoS}_{2}$ nanosheets.

Effect of concentration of $\mathrm{MoS}_{2}$ - Fig. 2a represents the effect of concentration of $\mathrm{MoS}_{2}$ nanosheets on codeposition of $\mathrm{MoS}_{2}$. The Ni$\mathrm{MoS}_{2}$ nanocomposite coatings are prepared by varying the concentrations of $\mathrm{MoS}_{2}$ in the plating bath $(0.5,1.0$ and $1.5 \mathrm{~g} / \mathrm{L})$ at constant current density of $2.5 \mathrm{~A} / \mathrm{dm}^{2}$ with stirring rate of $300 \mathrm{rpm}$. The amount of codeposition of $\mathrm{MoS}_{2}$ is found to be increased with increase in $\mathrm{MoS}_{2}$ concentration. A maximum codeposition of $15 \mathrm{wt} \%$ is observed for the bath having $1 \mathrm{~g} / \mathrm{L}$ concentration of $\mathrm{MoS}_{2}$ nanosheets. The maximum codeposition of $\mathrm{MoS}_{2}$ in the nickel matrix refers to the adsorption on the cathode surface which can be explained by Guglielmi's two-step adsorption model. ${ }^{25,26}$ At higher concentration (1.5 $\mathrm{g} / \mathrm{L}$ ), the amount of $\mathrm{MoS}_{2}$ codeposition slightly reduced due to saturation in adsorption on the cathode surface.

Effect of stirring rate - The relationship between the weight percentage (wt \%) of codeposited $\mathrm{MoS}_{2}$ and the stirring rate is shown in Fig. 2b. With increase in stirring rate, the codeposition of $\mathrm{MoS}_{2}$ is increased and reached a maximum value of 17 wt $\%$ in the Ni-MoS composite at $400 \mathrm{rpm}$. Further, increase in agitation rate reduces the codeposition due to collision factor. Another reason may be the increased streaming velocity of the bath sweep away the loosely bound particles from the cathode surface and make the rate of particle removal higher than the rate of adsorption in the cathode surface which results in a decrease in the amount of codeposited $\mathrm{MoS}_{2}$ in the nickel matrix. ${ }^{22}$ Thus, the stirring rate for maximum amount of codeposition of $\mathrm{MoS}_{2}$ is optimized and is found to be $400 \mathrm{rpm}$.

FE-SEM studies - Surface morphologies of the pure Ni and Ni$\mathrm{MoS}_{2}$ composite coatings are analyzed using FE-SEM and the results are shown in Fig. 3. The surface of pure Ni shows uniform structure with higher grain size. Ni-MoS 2 coating has uniform deposition with smaller grain. The addition of $\mathrm{MoS}_{2}$ in the nickel matrix has changed the microstructure of the coating. The addition of $\mathrm{MoS}_{2}$ nanosheets hinder the crystal growth and increases the nucleation sites for the reduction of $\mathrm{Ni}$ ions which results in reduced grain size. ${ }^{9,20}$

$X$-ray diffraction studies - The X-ray diffraction diagrams of pure $\mathrm{Ni}$ and $\mathrm{Ni}-\mathrm{MoS}_{2}$ composite coatings are shown in Fig. 4a. Three major peaks are visible for pure $\mathrm{Ni}$ as well as $\mathrm{Ni}-\mathrm{MoS}_{2}$ coatings. However, some differences in the intensity of (111), (200) and (220) peaks are observed in the composite coating. Two peaks of $\mathrm{MoS}_{2}$ are visible which can be attributed to (002) and (006) planes of hexagonal $\mathrm{MoS}_{2}$. The peaks of $\mathrm{MoS}_{2}$ are short due to high relative intensity of Ni peaks. The average crystallite size of $\mathrm{Ni}$ deposit was calculated using Scherrer formula (Eq. 1) and is shown in Table 2. From the table, it is evident that the incorporation of $\mathrm{MoS}_{2}$ has reduced the crystallite size of $\mathrm{Ni}$ in the composite coating.

The preferred crystal orientation of Ni was calculated using RTC values (Eq. 2) and the results are shown as bar graph in Fig. 4b. The preferred crystal orientation for the pure Ni coating is (220) with the maximum texture coefficient (TC) value of $41 \%$, whereas for $\mathrm{Ni}-\mathrm{MoS}_{2}$ coating, the preferred crystal orientation is (111) plane with the maximum TC value of $55 \%$. The incorporation of $\mathrm{MoS}_{2}$ in the $\mathrm{Ni}$ matrix inhibit the grain growth of $\mathrm{Ni}$ along its preferred direction and change the preferred orientation to different plane. ${ }^{27}$

Fig. 5 shows the Williamson-Hall plots of pure Ni and Ni-MoS composite coatings. The values of lattice strain and crystallite size are
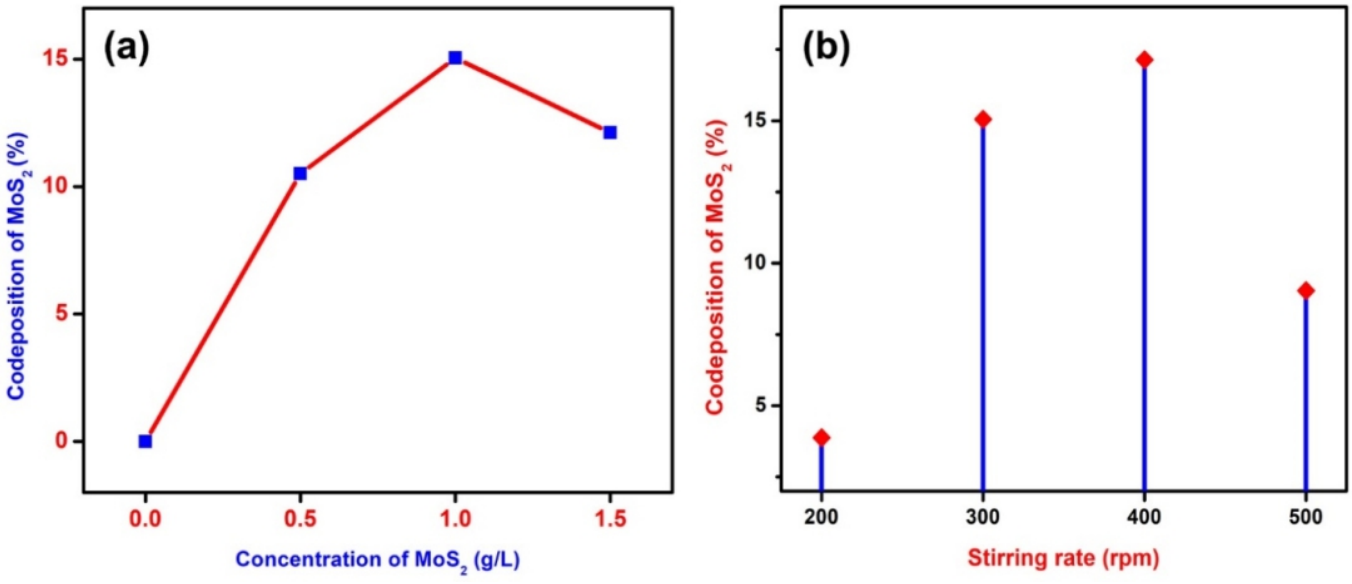

Fig. 2 (a) Effect of concentration of $\mathrm{MoS}_{2}$ on codeposition of $\mathrm{MoS}_{2}$, (b) Influence of stirring rates on codeposition of MoS ${ }_{2}$ nanosheets. 
Table 2 Scherrer's equation parameters for pure $\mathrm{Ni}$ and $\mathrm{Ni}-\mathrm{MoS}_{2}$ composite coatings.

\begin{tabular}{cccccc}
\hline Samples & (hkl) plane & $\theta$ & FWHM & D (nm) & Avg. D (nm) \\
\hline \multirow{2}{*}{ Pure Ni } & $(111)$ & 22.23 & 0.33584 & 25.26 & \\
& $(200)$ & 25.97 & 0.44768 & 29.51 & $\mathbf{2 4 . 6}$ \\
& $(220)$ & 38.25 & 0.52039 & 19.21 & \\
\hline \multirow{2}{*}{ Ni-MoS } & $(111)$ & 22.2 & 0.38366 & 22.1 & \\
& $(200)$ & 25.88 & 0.54038 & 16.15 & $\mathbf{1 8 . 7}$ \\
& $(220)$ & 38.17 & 0.55521 & 17.99 & \\
\hline
\end{tabular}
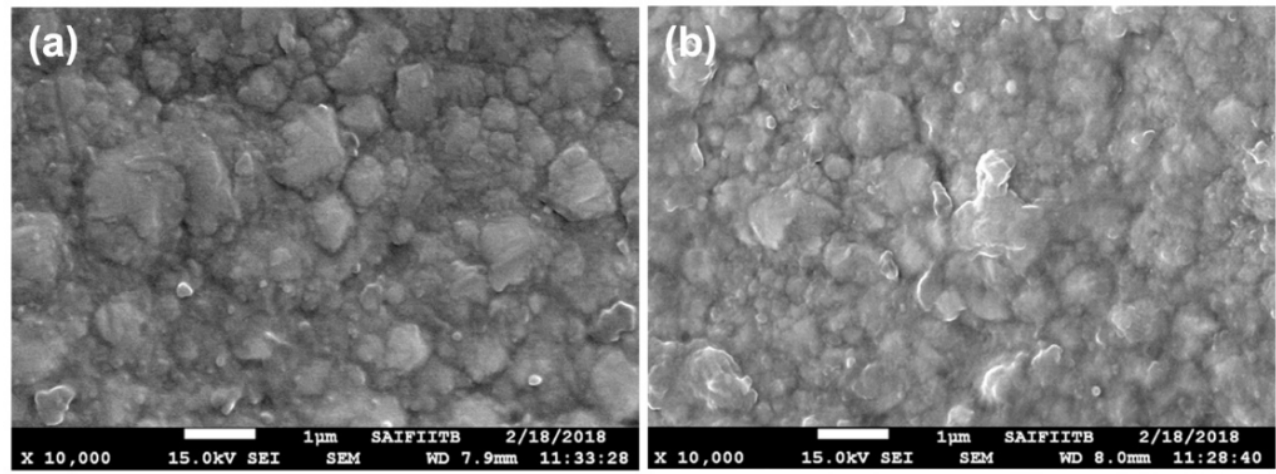

Fig. 3 FE-SEM images of (a) pure Ni, (b) Ni-MoS 2 composite coating.
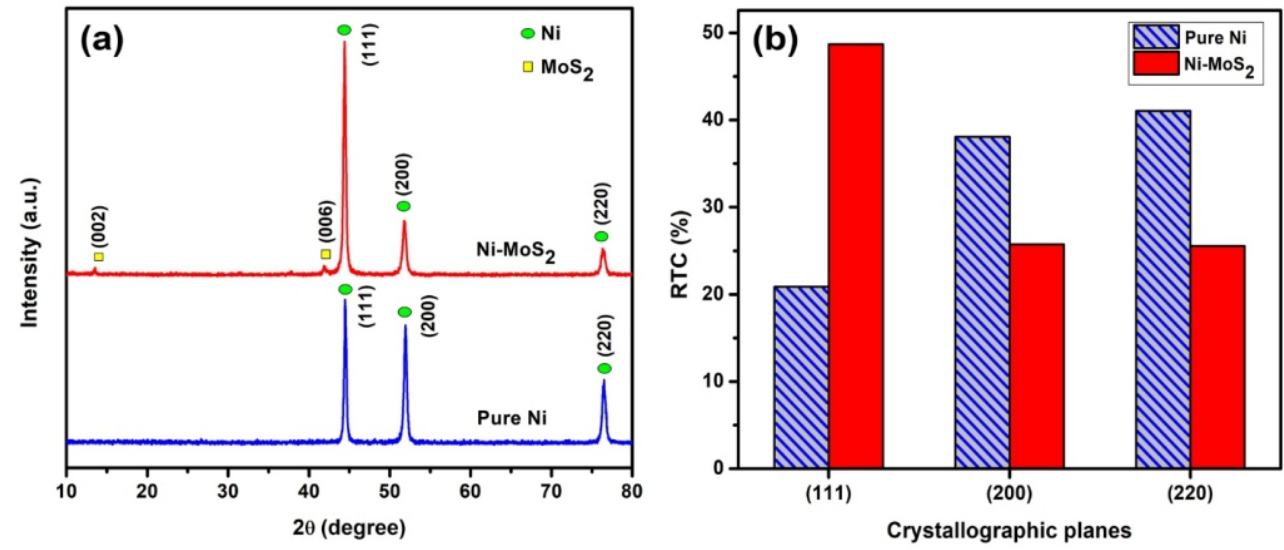

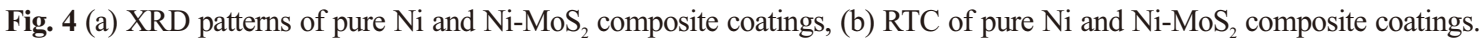
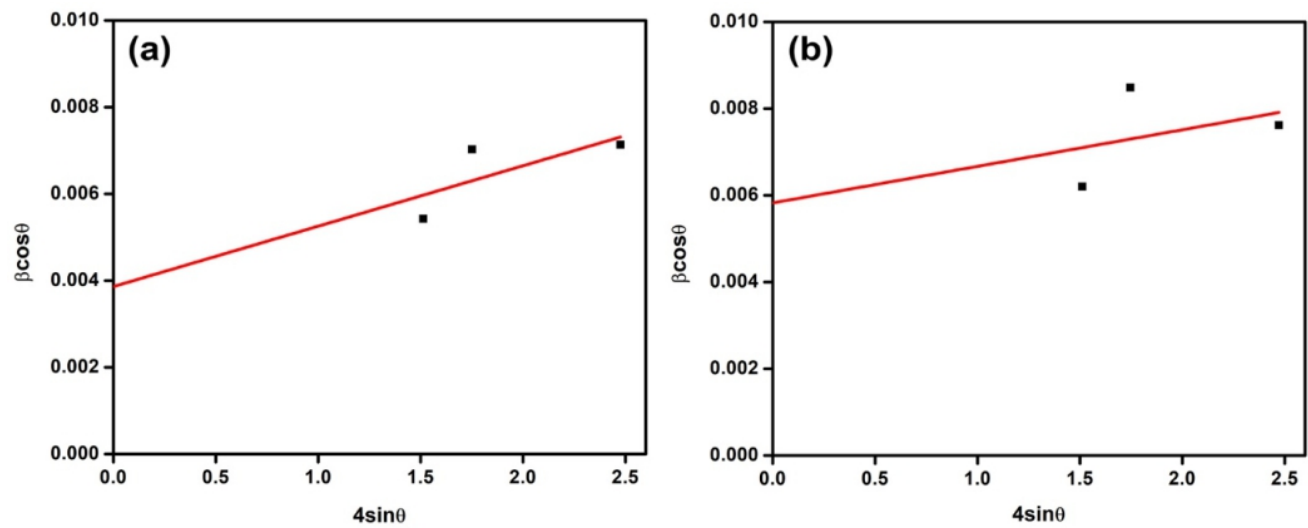

Fig. 5 Williamson-Hall plot for pure $\mathrm{Ni}$ and Ni-MoS 2 composite coatings 

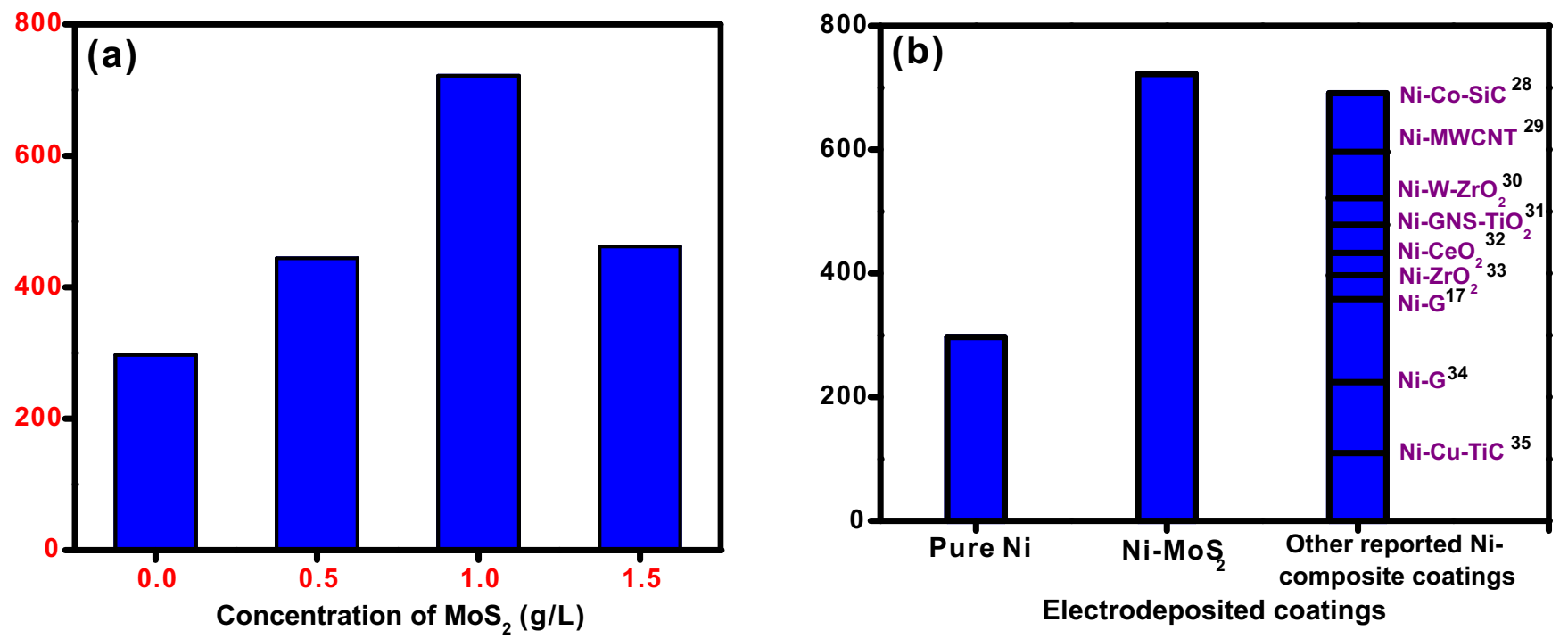

Fig. 6 (a)Microhardness of pure Ni and Ni-MoS 2 composite coatings at different concentration of $\mathrm{MoS}_{2}(0.5,1.0$ and $1.5 \mathrm{~g} / \mathrm{L})$ and (b) comparison of microhardness of Ni-MoS 2 composite coating with other reported Ni-composite coatings.

calculated from the plot and are given in Table 3. From the table, it can be seen that the average crystallite size of nickel has reduced with the addition of $\mathrm{MoS}_{2}$ in the Ni matrix. There is an increase in crystallite size obtained from Williamson-Hall method compared to crystallite size calculated using Scherrer's formula. This is due to the negligence of instrumental broadening error. The lattice strain calculated from the Williamson-Hall method is found to decrease for Ni-MoS $\mathrm{M}_{2}$ composite coating compared to pure Ni coating. This shows that there is a stress relaxation when $\mathrm{MoS}_{2}$ is added into the $\mathrm{Ni}$ matrix. The $\mathrm{MoS}_{2}$ nanosheets fill the defects and grain boundaries which result in grain boundary relaxation and release of stress.

Microhardness - The Vickers microhardness of pure Ni and Ni-MoS composite coatings obtained at various concentrations of $\mathrm{MoS}_{2}$ in the $\mathrm{Ni}$ matrix are shown in Fig. 6a. The microhardness of pure Ni is found to be $297 \mathrm{HV}$. It is tend to increase with the addition of $\mathrm{MoS}_{2}$ in the Ni matrix. At the concentration of $1 \mathrm{~g} / \mathrm{L}$ of $\mathrm{MoS}_{2}$, it shows the maximum microhardness value of $722 \mathrm{HV}$. This value is higher than the microhardness value of other electrodeposited $\mathrm{Ni}$ composite coatings

is shown in Fig. 6b. ${ }^{1728-35}$ At higher concentration of $1.5 \mathrm{~g} / \mathrm{L}$ of $\mathrm{MoS}_{2}$, the microhardness tend to decrease due to decrease in $\mathrm{MoS}_{2}$ content in the Ni matrix. The increase in microhardness can be attributed to the increase in codeposition of $\mathrm{MoS}_{2}$. The rise in $\mathrm{MoS}_{2}$ content in the Ni matrix has resulted in decrease in grain size. The $\mathrm{MoS}_{2}$ nanosheets adsorbed on the growth centers of $\mathrm{Ni}$ which inhibited the growth of nuclei by blocking the surface of growing $\mathrm{Ni}$ and thus increasing the rate of nucleation and decreasing the grain size of nickel. More the amount of $\mathrm{MoS}_{2}$ nanosheets, more the number of grains and grain. ${ }^{20}$ This grain refinement strengthening the material by blocking the dislocation motion and grain boundary sliding. ${ }^{36}$ The enhancement in microhardness is due to grain refinement that can be explained by the Hall-Petch relation. The experimental result confirms the validation of Hall-Petch effect. But, the validation of HallPetch relation and previous studies confirm that grain size has reduced with increase in codeposition of $\operatorname{MoS}_{2}{ }^{9,22}$

Wear resistance - The wear weight loss of pure Ni and Ni-MoS composite coatings are calculated by measuring weight of the test samples before and after the wear test. The weight loss was found to be $5.2 \mathrm{mg}$ for pure $\mathrm{Ni}$ and $2.8 \mathrm{mg}$ for $\mathrm{Ni}-\mathrm{MoS}_{2}$ composite coating. The graph between coefficient of friction versus wear test time for electrodeposited pure $\mathrm{Ni}$ and $\mathrm{Ni}-\mathrm{MoS}_{2}$ composite coating is shown in Fig. 7a. The friction coefficient exhibits a lower value for $\mathrm{Ni}-\mathrm{MoS}_{2}$ coating compared to pure Ni. For pure $\mathrm{Ni}$ coating, the friction coefficient shows a steady increase at first, then comes down to a lower value and keeps stable at $\sim 0.5$ with prolonged wear test time. Ni-MoS coating has a gradual increase to a friction coefficient value of $\sim 0.4$, but shows a fluctuation thereafter and again picks up to a steady value of 0.4. The wear loss and friction coefficient is calculated for pure $\mathrm{Ni}$ and Ni-MoS ${ }_{2}$ composite coating is shown in Fig. 7b. The Ni-MoS shows a higher wear resistance than the Ni coating. Hence the friction coefficient value of $\mathrm{Ni}-\mathrm{MoS}_{2}$ composite coating is lower than the other

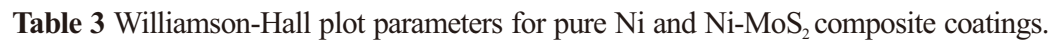

\begin{tabular}{ccc}
\hline Samples & Lattice strain, $\varepsilon$ & Cry stallite size, D (nm) \\
\hline Pure Ni & $1.39 \times 10^{-2}$ & 35.5 \\
$\mathrm{Ni}-\mathrm{MoS}_{2}$ & $8.43 \times 10^{-4}$ & 23.5 \\
\hline
\end{tabular}



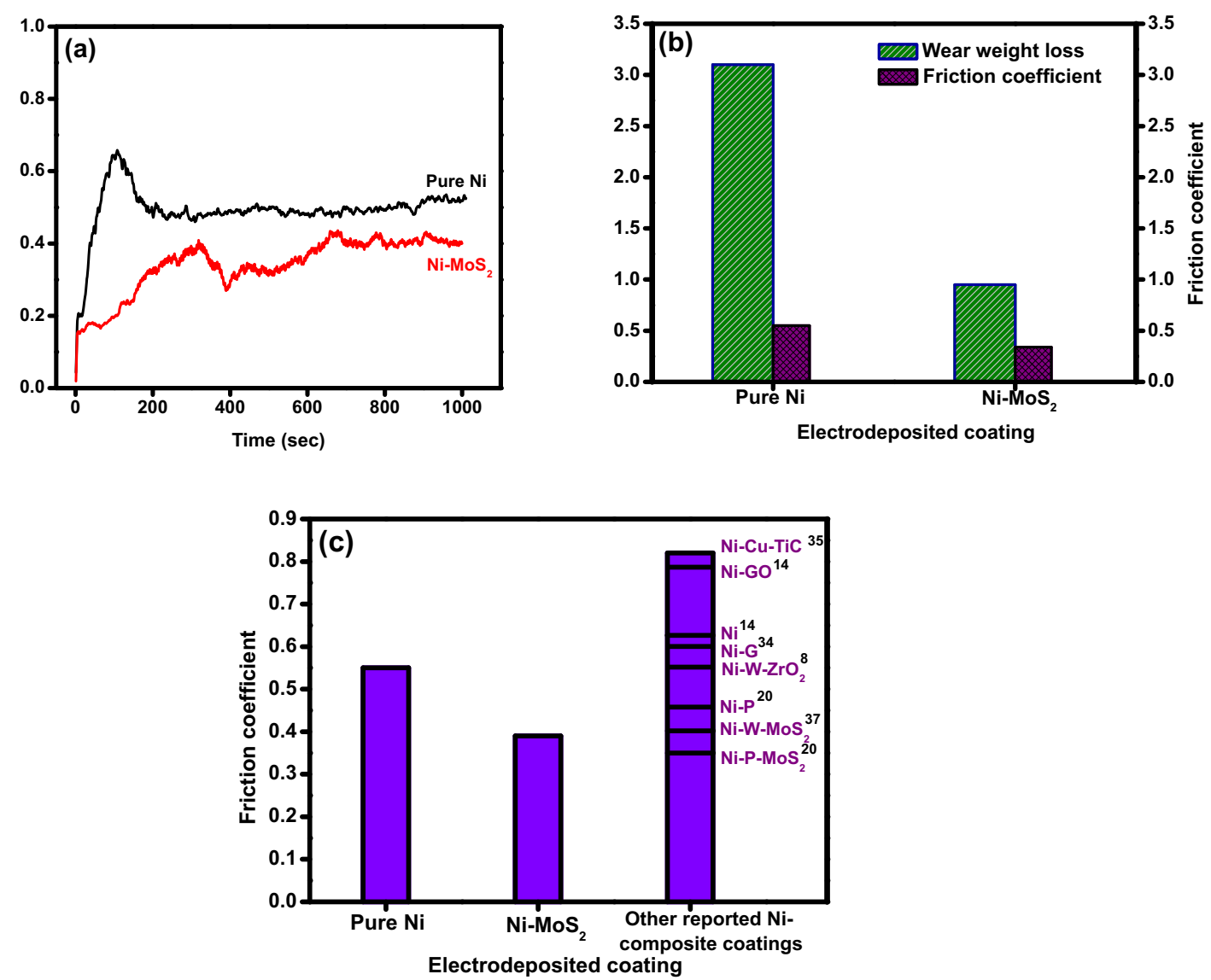

Fig. 7 (a) Friction coefficient vs wear test time for pure Ni and Ni-MoS ${ }_{2}$ composite coatings, (b) Wear weight loss and friction coefficient of pure Ni and Ni-MoS ${ }_{2}$ composite coatings and (c) Comparison of friction coefficient of Ni-MoS $\mathrm{S}_{2}$ composite coating with other reported Ni-composite coatings.

electrodeposited Ni composite coatings is shown in Fig. 7c. ${ }^{8,14,20,34,35,37}$ Moreover, the weight loss of $\mathrm{Ni}-\mathrm{MoS}_{2}$ composite coating is lower compared with reported literature. ${ }^{14,20,37}$ The addition of $\mathrm{MoS}_{2}$ nanosheets in the composite coating reduces the coefficient of friction and increases the wear resistance by sliding between the van der Waal's layers and act as a solid lubricant. In addition to friction coefficient, the grain refinement and microhardness also contribute to improve the wear resistance. ${ }^{38,39}$

\section{Conclusions}

$\mathrm{MoS}_{2}$ nanosheets were successfully synthesized by a facile hydrothermal method and confirmed its formation by XRD and FE-SEM analysis. $\mathrm{MoS}_{2}$ nanosheets were codeposited in the Ni matrix using direct current electrodeposition method and the maximum codeposition of $\mathrm{MoS}_{2}$ was obtained at a concentration of $1 \mathrm{~g} / \mathrm{L}$ with the stirring rate of $400 \mathrm{rpm}$. The XRD and FE-SEM studies on the coatings confirmed that the addition of $\mathrm{MoS}_{2}$ inhibited the crystal growth and thereby changed the preferred crystal orientation and reduced crystallite size and lattice strain. The Ni-MoS $\mathrm{M}_{2}$ nanocomposite coating exhibited a maximum microhardness $(722 \mathrm{HV})$ due to grain refinement. Friction measurements produced better wear resistance for $\mathrm{Ni}-\mathrm{MoS}_{2}$ composite coating compared to pure Ni coating. These results revealed that Ni$\mathrm{MoS}_{2}$ composite coating can be used as a hard and wear resistant coating for solid lubricant applications.

\section{Acknowledgements}

The authors gratefully acknowledge the Central Instrumentation Facility
(CIF), Pondicherry University for providing the instrumentation facilities.

\section{Conflict of interest}

The authors declare that they have no conflict of interest

\section{References}

1. S. Ramalingam, K. Balakrishnan, and A. Subramania, Trans. IMF, 2015, 93, 262-266.

2. C. Ma, S. C. Wang, L. P. Wang, F. C. Walsh, and R. J. K. Wood, Surf. Coat. Technol., 2013, 235, 495-505.

3. K. M. Zadeh, R. A. Shakoor, and A. B. Radwan, Int. J. Electrochem. Sci., 2016, 11, 7020-7030.

4. K. A. Kumar, G. P. Kalaignan, and V. S. Muralidharan, Appl. Surf. Sci., 2012, 259, 231-237.

5. N. P. Wasekar and G. Sundararajan, Wear, 2015, 342-343, 340-348.

6. N. P. Wasekar, S. M. Latha, M. Ramakrishna, D. S. Rao, and G. Sundararajan, Mater. Des., 2016, 112, 140-150.

7. P. Baghery, M. Farzam, A. B. Mousavi, and M. Hosseini, Surf. Coat. Technol., 2010, 204, 3804-3810.

8. A. Bigos, P. Indyka, A. Chojnacka, A. Drewienkiewicz, S. Zimowski, M. Kot, and M. J. Szczerba, J. Electroanal. Chem., 2018, 813, 39-51.

9. T. Borkar and S. Harimkar, Surf. Eng., 2011, 27, 524-530.

10. H. Jin, Y. Wang, Y. Wang, and H. Yang, Rare Met., 2017.

11. S. Khabazian and S. Sanjabi, Appl. Surf. Sci., 2011, 257, 5850-5856.

12. C. T. J. Low, J. O. Bello, J. A. Wharton, R. J. K. Wood, K. R. Stokes, and F. C. Walsh, Surf. Coat. Technol., 2010, 205, 1856-1863.

13. N. S. Qu, D. Zhu, and K. C. Chan, Scr. Mater, 2006, 54, 1421-1425.

14. Z. Xue, W. Lei, Y. Wang, H. Qian, and Q. Li, Surf. Coat. Technol., 2017, 
325, 417-428.

15.Y. Zhang, X. Leng, X. Wang, P. Ou, and W. Zhang, Metallogr. Microstruct. Anal., 2017.

16. S. Ramalingam, K. Balakrishnan, S. Shanmugasamy, and A. Subramania, Surf. Eng., 2017, 33, 369-374.

17. C. M. P. Kumar, T. V Venkatesha, and R. Shabadi, Mater. Res. Bull., 2013, 48, 1477-1483.

18. B. Kirubasankar, V. Murugadoss, and S. Angaiah, RSC Adv., 2017, 7, 5853-5862.

19. A. Castellanos-gomez, M. Poot, G. A. Steele, and H. S. J. Van Der Zant, Adv. Mater., 2012, 24, 772-775.

20. Y. He, S. C. Wang, F. C. Walsh, Y. Chiu, and P. A. S. Reed, Surf. Coat. Technol., 2016, 307, 926-934.

21. S. Ott, P. G. Sanders, A. Borbe, J. R. Weertman, and T. Unga, Acta Mater., 1998, 46, 3693-3699.

22. R. Sen, S. Das, and K. Das, Surf. Coat. Technol., 2011, 205, 3847-3855.

23. M. Chhowalla and G. A. J. Amaratunga, Nature, 2000, 407, 164-167.

24. N. Wakabayashi, Phys. Rev. B, 1975, 12, 659-663.

25. N. Guglielmi, J. Electrochem. Soc., 1971, 1501, 1009-1012.

26. S. Ramalingam, V. S. Muralidharan, and A. Subramania, J. Solid State Electrochem., 2009, 13, 1777-1783.

27. A. G. Mccormack, M. J. Pomeroy, and V. J. Cunnane, J. Electrochem.
Soc., 2003, 150, 356-361.

28. L. Shi, C. Sun, P. Gao, F. Zhou, and W. Liu, Appl. Surf. Sci., 2006, 252, 3591-3599.

29. S. Khabazian and S. Sanjabi, Appl. Surf. Sci., 2011, 257, 5850-5856.

30. Q. Niu, G. Liu, and C. Ran, IOP Mater. Sci. Eng, 2018, 301, 12001.

31. M. W. Khalil, T. A. Salah Eldin, H. B. Hassan, K. El-Sayed, and Z. Abdel Hamid, Surf. Coatings Technol., 2015, 275, 98-111.

32. H. Jin, Y.-Y. Wang, Y.-T. Wang, and H.-B. Yang, Rare Met., 2018, 37, $148-153$.

33. G. Parida, D. Chaira, and A. Basu, Trans. Indian Inst. Met., 2013, 66, $5-11$.

34. J. Chen, J. Li, D. Xiong, Y. He, Y. Ji, and Y. Qin, Appl. Surf. Sci., 2016, 361, 49-56.

35. P. Jha, R. K. Gautam, and R. Tyagi, Friction, 2017, 5, 437-446.

36. H. Li, Y. He, T. He, Y. Fan, Q. Yang, and Y. Zhan, Ceram. Int., 2016, 42, 18380-18392.

37. M. F. Cardinal, P. A. Castro, J. Baxi, H. Liang, and F. J. Williams, Surf. Coatings Technol., 2009, 204, 85-90.

38. S. Shanmugasamy, K. Balakrishnan, A. Subasri, S. Ramalingam, and A. Subramania, J. Rare Earths, 2018, doi: 10.1016/j.jre.2018.06.004 .

39. Y. Fan, Y. He, P. Luo, T. Shi, and H. Li, J. Electrochem. Soc., 2015, 162, D270-D274. 